\title{
LOS VALORES ÉTICOS EN LA ESCUELA DIOCESANA DE ASISTENTES SOCIALES «SAN VICENTE DE PAÚL» DE PAMPLONA (1960-1985)
}

\author{
Ethical values at the Diocesan School of Social Work \\ «San Vicente de Paul» of Pamplona (1960-1985)
}

FRANCISCO IDARETA GOLDARACENA ${ }^{1}$

\section{Resumen}

El propósito del presente artículo consiste en estudiar el compromiso ético de las profesionales del Trabajo Social en la Escuela Diocesana de Asistentes Sociales «San Vicente de Paúl» de la Archidiócesis de Pamplona. Para ello, analizaremos la importancia que tuvo la Ética en la conformación de la profesión a través de los períodos comprendidos entre 1960 y 1985 utilizando una metodología histórico-sistemática. Durante este intervalo temporal, en España se identifican tres períodos fundamentales (Idareta, Úriz y Viscarret, 2016), mientras que en Navarra únicamente dos. En cada uno de ellos analizaremos el contexto histórico-filosófico $\mathrm{y}$ aquellos acontecimientos que pudieron sentar las bases del compromiso ético de las y los profesionales del Trabajo Social.

Palabras clave: Trabajo Social - Navarra - Ética profesional - Escuela de Asistentes Sociales - Valores éticos

\section{Extensive summary}

In a previous study (Idareta, Úriz and Viscarret, 2016) the periodisation of ethics in Social Work in Spain was established from 1861 until the present day. In this case, we will be concentrating on four of these: the stoicism period, the period of moralisation, the period of values and the period of ethical principles, bureaucratisation and demands. This is because, given the lack of studies of similar characteristics, we attempt to analyse the trajectory of the Diocesan College of Social Workers «San Vicente de Paúl» in Pamplona from an ethical perspective, as well as its classes between 1960 and 1985 . We have thus carried out an exhaustive bibliographical review and a historical-systematic in-depth study of the work of the most prominent authors on this matter.

First of all, we will analyse the periodisation of ethical values in Social Work in Spain and, subsequently, in Navarra.

In relation to the periodisation of ethical values in Social Work in Spain, Idareta, Úriz and Viscarret (2016)

\begin{abstract}
The purpose of this paper is to study the ethical commitment of female professional social workers at the Diocesan School of Social Work «San Vicente de Paul», attached to the Archdiocese of Pamplona. To this goal, we will discuss the significance of Ethics in shaping the profession through the periods between 1960 and 1985 using a historical-systematic methodology. Three main periods are identified in this time interval in Spain (Idareta, Úriz and Viscarret, 2016), while only two in Navarra. The authors will analyze the historical and philosophical context in each of these periods, as well as the events that could lay the foundation of ethical commitment from social work professionals.
\end{abstract}

Keywords: Social Work - Navarra - Professional Ethics - School of Social Work - Ethical Values

organises ethical values in Social Work in Spain into five periods. As already stated, on this occasion we will analyse four of those: the stoicism period, the period of moralisation, the period of values and the period of ethical principles, bureaucratisation and demands.

In the first period (1861-1936), the influence of stoicism on the Social Worker is evident, whether this is due to Spanish Krausism or contributions from the likes of Concepción Arenal (Lacalzada, 2012).

Therefore, in 1932 in the middle of the II Republic, Raul Roviralta and Antonia Ferreras founded the first College of Social Workers in Barcelona, named the «College of Social Work for Women» which, in its beginning «disregarded all official concrete faith as the political circumstances encouraged this» (Molina, 1994, 86).

In the curriculum for the 1932-1933 academic year it starts with a class in Moral Philosophy which emphasised the importance of the «technification» of social work, as well as faith-based tendencies (Vázquez, 1971).

Recibido: 10/10/2016

Aceptado: 05/12/2016

Publicado: 20/12/2016

1. Universidad Pública de Navarra. Departamento de Trabajo Social. Edificio Departamental de las Encinas. Campus Arrosadía (31006). Pamplona. Tfno.: 948169693.

Este artículo ha sido financiado por el Ministerio de Economía y Competitividad del Gobierno de España en el marco del proyecto de investigación FFI2013-46530-P: Toma de decisiones éticas en la práctica profesional del Trabajo Social. 
The moralisation period (1939-1960) is characterised by exacerbated paternalism. After the coup d'état by Franco (1936), the State was subject to substantial international isolation which definitely constrained the development of Social Work in this era (Sarasa, 1993). In this context, the College of Social Work of Barcelona presented itself as being openly faith-based, giving way for primary tensions among its supporters.

The relationship between professional morality and religion is evident in that, years later, the majority of professionals at the national level moved away from religion (Llovet and Usieto, 1990).

Due to the increase of colleges, we will be highlighting the creation of the College of Social Work «San Vicente Paúl» in 1957 in Madrid. The College aimed to provide training to the Daughters of Charity Congregation which at that time was developing its apostolic and social work in the social work sector.

In turn, in 1958, the slogans publicised by the colleges started to echo the emerging though faint axiological renewal of the profession. Even the study plans for this same year from the Spanish Federation of Community Services Colleges of the Church (FEISS) take on the professional morality which is removed from religion.

During the period of values (1960-1980) an important renovation of professional values can be observed. The first sub-period of retreat towards an axiological basis for professional identity (1960-1965) is one of the most significant periods in Social Work from an axiological point of view. In 1960, the Diocesan College of Social Work «San Vicente de Paul» was created in Pamplona and the first class of the College of Social Work «San Vicente Paul» of Madrid was set up.

We continue to find evidence of professional morality related to religious training. In this era the religious character of the profession was predominant but less and less so. In the second sub-period of ethical reflexion (1965-1970) the celebration of the $1^{\text {st }}$ National Congress in Barcelona is noted as the lack of a deontological code of conduct, which ethically guarantees the specific form of intervention, was highlighted.

The third sub-period (towards the axiological emancipation of professional identity) (1970-1980) is characterised by the evidence that professional morality once again was dissociated from religious training. It is clear that, from an axiological point of view, this is the moment in which professionals started to become aware of their own ethical space.

Therefore, although there were still professionals with a clear religious character, in this period the number of professionals who believed that their professional ethics should be disassociated from religious morality were on the increase (Vázquez, 1971).

In the period of ethical principles, bureaucratisation and demands (1980-2002), the phenomenon which was most prominent in Social Work was that of bureaucratisation and the subsequent depletion of the discipline-profession as well as the demands of social work professionals who took on leftist ideologies and refused to identify the profession with religious values (author, 2016).

In regards to the periodisation of ethical values in Social Work in Navarra, we have established two periods between 1960 and 1985: the moralisation period (1960-1980) and the period of ethical principles, bureaucratisation and demands (1980-1985). The first is linked to the decline of the moralisation period which originated at the national level while in Navarra the continuity of this period was longer. The second coincides with the period of the same name that took place on a national level in 1980 which saw the emergence of the secularisation of Social Work in Navarra.

In the moralisation period (1960-1980), the College addresses the Archbishopric of Pamplona. In this sense, in the curriculum of the College of Pamplona for the 19621963 academic year, the class on Professional Morality is taught in the third year and is probably preconditioned by religious precepts taken from classes on Dogma and Sacred Writings from the first course or Social Doctrine of the Church from the second course (Romero, 2015). Humanising treatment and strong ethical sense appear as common distinctive features of both the first students of social work and teachers (Molina 1990, 1994).

All of this shows that Social Work in Navarra, as throughout the rest of Spain, started to be undertaken without any concrete method and without questioning the dominant hegemonic discourse (Romero, 2015; Idareta, Úriz and Viscarret, 2016), which may have compensated the important absence of technical orientation with a strong ethical orientation. A large number of testimonies from the era point to ethical values being fundamental in professional social work given the lack of a specific methodology in Social Work (Llovet and Usieto, 1990).

In the period of ethical principles, bureaucratisation and demands (1980-1985) the secularisation of Social Work in Navarra is highlighted, starting from the end of the 1970s (Campo and Celaya, 1981) with the gradual abandonment of voluntary practices and the subsequent professionalization of social intervention. In 1980, however, the College aspired to depend less on the Archbishopric and more on the Regional Government. All of this was because at the end of the 1970's, the Archbishopric threatened the closure of the College due to economic reasons and those of social change.

The College of Social Work «San Vicente de Paul» disappeared between 1984-1985, 25 years since its creation, to give way to the University College of the Government of Navarra. Therefore, in 1984, the definitive steps towards a university standard of Social Work in Navarra were taken, attached to the University of Zaragoza until 1991. It was incorporated into the Public University of Navarra afterwards.

\section{Conclusions}

From an ethical perspective, the case of Social Work in Navarra differs substantially from the subsequent development in Spain as a whole. While at the national level, between 1960 and 1985 there are three clearly defined ethical periods (the moralisation period, the period of values and the period of ethical principles, bureaucratisation and demands) (Idareta, Úriz and Viscarret, 2016), in Navarra, only two can be identified (the moralisation period and the period of ethical principles, bureaucratisation and demands)

In light of the above, we can affirm that while in most of Spain, the process of secularisation of social work colleges begins in the 1960's, in Navarra it begins in the late 1970's and early 1980's. Therefore, in Navarra, the moralisation period lasts for a further 20 years while throughout the rest of Spain, between 1960 and 1980, the period of values was already underway. 


\section{Introducción}

En un estudio anterior (Idareta, Úriz y Viscarret, 2016) se estableció la periodización de los valores éticos del Trabajo Social en España desde 1861 hasta la actualidad. En esta ocasión, nos centraremos en cuatro de ellos: en el período de influencia del estoicismo, en el período moralizador, en el período de valores y en el período de principios éticos, burocratización y reivindicaciones. Todo ello porque pretendemos analizar desde una perspectiva ética la trayectoria de la Escuela Diocesana de Asistentes Sociales «San Vicente de Paúl» de Pamplona y la de sus promociones entre 1960 y 1985.

Según Idareta, Úriz y Viscarret (2016), aunque a nivel nacional el período de valores hunde sus raíces en el período moralizador, comienza a desvincularse de él tomando como referencia valores propios del período de influencia del estoicismo. De ahí la importancia de analizar los cuatro y de destacar la particularidad del primer subperíodo de valores (1960-1965) en el que, a nivel nacional, se inician los primeros intentos renovadores de la profesión a la par que se retorna a valores propios del estoicismo en la profesión.

Por su parte, la Escuela Diocesana «San Vicente de Paúl» surge en 1960 y, debido principalmente a su dependencia institucional, estuvo muy condicionada por los valores cristianos hasta su clausura. Como veremos a continuación, a nivel nacional el período moralizador se inicia en 1939 y comienza a remitir a finales de los años 50 e inicios de los 60.

En el caso de Navarra, se pone de manifiesto que la Escuela surge en pleno declive del período moralizador a nivel nacional y que, lejos de seguir la trayectoria nacional, se originará una prolongación de este período moralizador hasta finales de los años 70. Momento a partir del cual seguirá la misma evolución que el resto de comunidades autónomas.

Por todo ello, en primer lugar analizaremos la periodización de los valores éticos del Trabajo Social en España y, posteriormente, en Navarra.

\section{Periodización de los valores éticos en el Trabajo Social en España}

Idareta, Úriz y Viscarret (2016) organizan en cinco períodos los valores éticos del Trabajo Social en España. Como ya lo anticipáramos, en esta ocasión analizaremos cuatro de ellos: el período de influencia del estoicismo, el período moralizador, el período de valores y el período de principios éticos, burocratización y reivindicaciones. 


\subsection{Período de influencia del estoicismo (1861-1936)}

Según Zambrano $(1992,1996)$, el estoicismo es aquella síntesis de toda la filosofía griega a la que le preocupa el desamparo del ser humano y a la que se recurre cuando se atraviesa una crisis y cuando las ideas religiosas son incapaces de dar respuesta a nuestras preguntas existenciales. El estoicismo «ha significado, en efecto, el lado laico de nuestra cultura» $\mathrm{y}$ «constituye el mayor intento de vivir según la filosofía» (Zambrano, 1996: 58-59).

En España el estoicismo ha convivido con el cristianismo en diferentes épocas, enlazando perfectamente con él (Ganivet, 1998; Domínguez, 2011). De hecho, se lo ha considerado como corriente de pensamiento que ha precedido al cristianismo. Concretamente, el estoicismo senequista encajó perfectamente tanto con el krausismo español como con la moral cristiana (Díaz García, 1985; Fernández y Corvalán, 2001) y pese a sus notables diferencias, en algunos contextos históricos, el cristianismo ha estado inspirado en el estoicismo (Domínguez, 2011).

Destacaremos las aportaciones de Juan Luis Vives y de San Vicente de Paúl como precursores del Trabajo Social.

Juan Luis Vives (1492-1539) en su ética humanístico-cristiana, mezcla el platonismo, el estoicismo y una profunda fe cristiana. Concretamente en De Disciplinis (1531) propone una profunda reforma del sistema educativo al concebir que la formación moral de cada persona es la única que asegura una auténtica reforma social. En 1524 escribe un breve manual sobre ética, Introductio ad sapientiam, en el que más nítidamente se mezclan cristianismo y estoicismo y en el que destaca la importancia que otorga a la praxis ética frente a los aspectos de índole formal (Domínguez, 2011).

Para San Vicente de Paúl (1570-1660) las aportaciones de Vives fueron inspiradoras en su defensa de los pobres y de los niños. Según San Vicente, ante las dificultades, la ecuanimidad y el equilibrio son fundamentales (Motto, 2011). La atención a las personas en situación de necesidad debía ofrecerse de forma individualizada. Estos y otros valores fueron perfectamente encarnados por la Congregación de Hermanas de la Caridad que Vives fundó, convirtiéndose así en las precursoras de las visitadoras sociales.

Años más tarde, de entre tales visitadoras sociales, destacaría Concepción Arenal, quien en su obra «La beneficencia, la filantropía y la caridad» (1861), diferencia tres conceptos: la beneficencia, que Arenal entiende como responsabilidad de los poderes públicos en la justa ordenación y organización de la sociedad en aras de ayudar al necesitado; la filantropía, que ella asemejaba a la conciencia de dignidad y del derecho de toda persona y al sentimiento de compasión por amor a la humanidad; y, finalmente, la caridad, que la entiende 
como compasión cristiana por amor a Dios y al prójimo. Fue una obra fundamental en la historia del Trabajo Social.

En este período, la influencia de valores estoicos en el incipiente Trabajo Social es evidente, bien sea a través del krausismo español, así como a través de aportaciones como la de Concepción Arenal (Lacalzada, 2012).

Así, en 1932, en plena II República, Raul Roviralta y Antonia Ferreras fundan en Barcelona la primera Escuela de Asistentes Sociales, denominada «Escuela de Asistencia Social para la Mujer». En su primer curso, la Escuela ofrecía «cultura femenina general orientada hacia los deberes cívicos y morales», siendo la orientación de la escuela «eminentemente humana y científica» (Vázquez, 1971: 42-43) y en los planes de estudio se reconocía que «la labor escolar tiene dos aspectos: uno de orden moral, el estudio de los problemas de justicia, y otro aspecto de orden técnico» (Vázquez, 1971: 43).

En aquel momento «se prescindió de toda oficialidad confesional concreta, ya que las circunstancias políticas así lo recomendaban» (Molina, 1994, 86). No debemos olvidar el profundo anticlericalismo que existía en aquella época (Sarasa, 1993) y, precisamente por ello, es probable que no estuviesen bien vistas ni las pretensiones ni los valores que profesaban las instituciones benéficas católicas, que eran la mayoría de las existentes.

En el programa de estudio del curso de 1932-1933 comienza a impartirse la asignatura de Filosofía Moral y destaca la importancia que se le otorga a la tecnificación de la asistencia social, así como la tónica confesional, que «ha marcado durante muchos años el contenido de los estudios de los asistentes sociales, el desarrollo de las escuelas de servicio social y, sobre todo, orientación de las actividades profesionales» (Vázquez, 1971: 39).

\subsection{El período moralizador (1939-1960)}

A partir de golpe de Estado franquista, se somete al Estado a un importante aislamiento internacional que condicionó definitivamente la evolución del Trabajo Social en esta época (Sarasa, 1993).

En dicho contexto, la Escuela de Asistente Sociales de Barcelona se posiciona abiertamente confesional, dando lugar así a las primeras tensiones entre sus promotores. Por una parte, la Escuela se ve obligada a abandonar su sede original, ya que el doctor Raúl Roviralta abandona la Escuela y al ser propietario del local, ésta tuvo que cambiar su sede, pasando a ser patrocinada por la Junta Diocesana de Mujeres de Acción Católica.

Por otra parte, la Escuela se ve abocada a tener que decidir entre una nueva sede para la Escuela o sus valores, aconfesionales hasta el momento, ya que esta Junta tenía como objetivo principal una enseñanza dirigida al apostolado, 
mientras que J.M. ${ }^{a}$ Llovera, que asumía la dirección en esta época, veía la necesidad de una preparación profesional libre de valores religiosos.

En 1937 el doctor Roviralta pública Los problemas de asistencia social en la Nueva España, donde señala que el valor fundamental de la asistencia social es la caridad y concreta un plan de estudios para la Escuela inspirándose en el de la Escuela Superior Fascista de Asistentes Sociales de Roma de 1927.

En los eslóganes publicitarios de la Escuela de Barcelona de 1939 se evidencia el perfil del alumnado al que se dirigían: «a todas las que aspiran a hacer el bien, a ser útiles, a las que seriamente quieren preparar su porvenir de esposas, madres de familia y madres espirituales de un mundo donde abundan los humildes, los ignorantes, los que sufren sin pan y sin verdad» (Molina, 1994: 145).

En 1940 se funda en Madrid la Escuela de Formación Familiar y Social con un carácter eminentemente confesional (Llovet y Usieto, 1990; Vázquez, 1971) y un año más tarde se intensificará el control de la moralidad del alumnado tras reconvertirse en Escuela Católica de Enseñanza Social, explicitando en el Artículo 40 de su reglamento que la Escuela «estará condicionada a la presentación por una persona conocida que responda de la moralidad de la aspirante».

El eslogan publicitario de la Escuela de Madrid de 1946 resulta especialmente elocuente a este respecto, ya que era una «carrera de los tiempos actuales al servicio de la Sociedad y de la Iglesia» cuya finalidad extraprofesional era «constituir con perfecta capacidad y responsabilidad un hogar cristiano» y la profesional dotar de herramientas en una carrera de «gran utilidad social y claro porvenir profesional» (Molina, 1994: 145).

Durante el primer curso 1942-1943, en la Escuela de Madrid se imparten las asignaturas de Educación religioso-moral, Cultura religiosa e Introducción a la moral profesional; mientras que en el segundo curso Clase religioso-moral, Cultura religiosa y Moral profesional. En el programa de estudios de 1949 se recoge la asignatura Dogma: Moral general y Moral profesional (Molina, 1994).

Se evidencia así la vinculación de la moral profesional con la religión de la que años más tarde se desmarcará la gran mayoría de profesionales a nivel nacional (Llovet y Usieto, 1990).

Del mismo modo que lo señalara Roviralta (1937), en 1946 Fernández de Henestrosa destacará que la caridad continuaba siendo el valor fundamental de la asistencia social.

En 1953 se crea, por una parte, la primera Escuela Masculina de Asistentes Sociales promovida por la Organización Católica y Profesional del Dependiente, así como la Escuela de Visitadoras Sociales Psiquiátricas, al amparo de la 
Sección Femenina de La Falange Española y de las JONS y el catedrático de psiquiatría Ramón Sarró.

Por la proliferación de escuelas a las que dio lugar, destacaremos la creación en 1957 de la Escuela de Asistentes Sociales «San Vicente Paúl» en Madrid. La Escuela pretendía dar formación a la Congregación de Hijas de la Caridad, que venía desarrollando su labor apostólica y social en el campo de la asistencia social, siendo, según Hörler, «las verdaderas precursoras de las asistentas sociales» (1961: 263).

Esta Escuela fue fundada por el Cardenal Prefecto Valerio Valeri y dirigida por la hermana sor Beatriz Gil Lansaque. La primera promoción de la Escuela de Asistentes Sociales «San Vicente Paul», que comienza sus estudios en 1957, se graduó en 1960.

La creación en 1957 de la Sección Social de Cáritas Nacional contribuye del mismo modo a la renovación axiológica de la profesión, ya que la entidad tiene como objetivo superar la tradicional acción benéfica que caracterizaba a la asistencia franquista. De hecho, fue gracias a Cáritas que se introdujo en España una nueva concepción de la asistencia social mucho más crítica con las injusticias sociales.

A su vez, en 1958 los eslóganes publicitarios de las escuelas comienzan a hacerse eco de la incipiente aunque tenue renovación axiológica de la profesión. La Escuela Católica de Enseñanza Social de Barcelona, recoge en su publicidad que «el trabajo social es una profesión que basándose en el conocimiento del hombre de la sociedad: ayuda a las personas, grupos y comunidades a participar en su promoción; actúa con las personas y grupos en situaciones de carencia debido a desajustes personales o deficiencias estructurales y sociales» (Molina, 1994: 147).

Incluso los planes de estudio de este mismo año de la Federación Española de Escuelas de la Iglesia de Servicio Social (FEISS) recogen la moral profesional desvinculada de la religiosa, ya que, a diferencia de los planes de estudios anteriores tanto de esta como del resto de escuelas, en el primer curso se oferta Cultura religiosa, Introducción a la moral profesional; y en el segundo curso Cultura religiosa, Moral profesional y Doctrina social de la Iglesia.

Todos estos elementos tienden a modificar el perfil profesional, así como la ideología de las y los profesionales. Había que luchar contra las desigualdades sociales pero sin paternalismos, seriamente enquistados en el ejercicio profesional (Llovet y Usieto, 1990). 


\subsection{Período de valores (1960-1980)}

Como veremos, durante este período se origina una importante renovación de los valores profesionales.

\subsubsection{Período de repliegue hacia la fundamentación axiológica de la} identidad profesional (1960-1965)

Este es uno de los períodos más significativos para el Trabajo Social desde el punto de vista axiológico. Pese a que en 1960 los recursos del Fondo Nacional de Asistencia Social eran todavía escasos y los servicios sociales continuarán siendo discrecionales y faltos de coordinación, es el año en el que se crea la Escuela Diocesana de Asistentes Sociales «San Vicente de Paúl» de Pamplona y se gradúa la primera promoción de la Escuela de Asistentes Sociales «San Vicente Paul» de Madrid.

En 1961 se constituye en España el Comité Nacional de Servicio Social y su representación ante las organizaciones internacionales y en 1962 se produce un avance relevante en la formación del Trabajo Social: junto con la unificación de las asociaciones de asistentes sociales, se acuerda que en el título no figure el carácter de confesionalidad católica, pese a que deba hacerse constar en algún apartado de los estatutos (Gil Parejo, 2004). Algo que, como vimos, ya se tuviera en cuenta en la elaboración de los estatutos de la primera escuela de Barcelona en 1935.

Otros dos hitos importantes en la historia del Trabajo Social en España fueron la creación en 1963 de la Federación Española de Asociaciones de Asistentes Sociales (FEDAASS) y la entrada en vigor en 1964 del Decreto 1403. La FEDAAS se creó bajo el amparo de la Iglesia y tuvo como propósito principal constituir un colegio de asistentes sociales en un futuro cercano, siendo Benita Llopis su primera presidenta. Por su parte, el Decreto 1403 regulaba y reconocía oficialmente los estudios para la formación de asistentes sociales en España. Gracias a este Decreto, la Administración reconoce a todas las escuelas de Trabajo Social.

El plan de estudios, aprobado por Orden Ministerial ${ }^{2}$, incluía en el primer curso Formación religiosa y Formación del Espíritu Nacional, en el segundo curso Formación religiosa. Doctrina Social de la Iglesia y Formación del Espiritu Nacional y en el tercer curso Formación religiosa. Moral Profesional. Volvemos

2. Orden Ministerial de 31 de Julio de 1964, en BOE 12/8/1964, ratificado por la Orden Ministerial de 26 de Agosto de 1966, BOE 1/12/1966). 
a encontrar un retroceso en los planes de estudio, ya que nuevamente aparece la moral profesional vinculada a la formación religiosa.

Comienzan a adquirir relevancia la autosuficiencia, el empoderamiento de la persona usuaria, la supresión de la emoción y el sentimiento en la intervención social, el secreto profesional como deber de la asistenta social, así como la denuncia expresa de la escasez de formación ética y la importancia de la ética profesional (Hörler, 1961).

No obstante, en este período también se confunde la moral profesional con la moral cristiana. De hecho, según Hörler (1961), el servicio social religioso o católico es el que ofrecía formación ética y ética profesional, mientras que el servicio social laico carecía de la misma o en escasa proporción.

En esta época todavía predomina el carácter religioso del ejercicio profesional, pero comienza a ser menos frecuente. De hecho, según Vázquez (1971), comienza a concebirse que la ayuda se sustenta sobre la tecnificación y el rigor científico de la asistencia social, trascendiendo las concepciones de beneficencia. Según Gutiérrez Resa (2010) los valores más importantes en los Servicios Sociales de esta época fueron la caridad, la solidaridad y la generosidad.

\subsubsection{Reflexión ética (1965-1970)}

En el eslogan publicitario de la Escuela de Asistentes Sociales de Vitoria de 1966 se indica que ésta «conjuga en sus enseñanzas cíclicas directrices morales y técnicas modernas sobre la persona en la sociedad», con «espíritu cristiano, femenino y social» (Molina, 1994: 146). Se comprueba la tendencia hacia la emancipación axiológica de la profesión, aunque sin poder lograr despegarse definitivamente de la moral cristiana.

En 1968 se celebra el I Congreso Nacional en Barcelona, donde se abordaron los siguientes temas: la terminología profesional (se sustituye el término de Asistencia Social por el de Trabajo Social con el propósito de reflejar una perspectiva de la disciplina técnica y profesional, en consonancia con las nuevas corrientes de América Latina y de los países anglosajones), las funciones profesionales, la formación y el código de ontológico.

Como señala Gil Parejo (2014: 16), «se constata la falta de un código deontológico que asegure éticamente la forma específica de actuar». Concretamente, en la cuarta y última ponencia, Montserrat Colomer plantea «la necesidad de confeccionar un Código de Deontología profesional para los asistentes sociales, adaptado a la realidad española, aceptándose en las conclusiones del congreso la redacción de un Código de Deontología que sirviera de base para el ejercicio profesional» (Gil Parejo, 2004: 23-24). 
Las y los profesionales comienzan a tomar conciencia de la importancia de la ética en su ejercicio profesional.

1.3.3. Hacia la emancipación axiológica de la identidad profesional (19701980)

En 1970 el Consejo General del Trabajo Social entra a forma parte como miembro de la FITS. Por estas fechas, existen en España 42 escuelas de servicio social reconocidas: el 69,1\% de las escuelas depende de la Iglesia, el 21,4\% es de dependencia estatal y paraestatal y el 9,5\% depende de entidades privadas (Vázquez, 1971).

La moral profesional vuelve a desvincularse de la formación religiosa. De hecho, en su investigación, Vázquez (1971) recoge que la opinión sobre la calidad de la docencia de la asignatura Formación Religiosa fue excelente para un $22,6 \%$ y buena para un 38,1\%, mientras que en el caso de la asignatura Moral Profesional para un $11,9 \%$ fue excelente y para un $47,6 \%$ fue buena.

No obstante, resulta revelador que las asignaturas de Formación en Espíritu Nacional y Formación Religiosa fueran consideradas por el alumnado como «suprimibles por innecesarias» (Vázquez, 1971: 110). Las propias alumnas parecen tener claro que estas asignaturas no encajan con sus valores profesionales.

En este sentido, en 1972 la Federación Internacional de Trabajadores Sociales (FITS) señala que los valores del Trabajo Social son la dignidad de toda persona, el desarrollo personal, la capacidad de las personas para la autodeterminación y la responsabilidad social.

Tanto en el II Congreso Nacional de Trabajo Social celebrado en Madrid en 1972, como en el III Congreso Nacional de Trabajo Social celebrado en Sevilla en 1976, queda patente la influencia del movimiento de reconceptualización latinoamericano en España, identificándose el marxismo como base teórica del mismo.

Concretamente en la mesa titulada «Trabajo Social y Ética Profesional» del III Congreso Nacional de Sevilla, los seis asistentes al grupo sobre Ética profesional hacen constar que no elaboraron conclusiones por el escaso número de participantes: «la ética profesional preocupó a menos del 1\% de las profesionales inscritas» (Congreso Nacional de Sevilla, 1976: 377).

Como veremos más adelante, en las III Jornadas Nacionales de Asistentes Sociales celebradas en Pamplona en 1976, se evidencia el interés de la profesión por «abandonar la actitud paternalista y construir una identidad profesional sólida» (Gil Parejo, 2004: 46), partiendo para ello de aquellos principios, metodología y ética del Trabajo Social heredados (De las Heras, 2000). 
Las Jornadas de Pamplona han sido consideradas como el inicio de la tecnificación profesional y el desarrollo disciplinar en el que, acorde con el movimiento de reconceptualización, se origina la ruptura con planteamientos clásicos y el alejamiento de posturas ideologizadas (Brezmes, 2008).

En 1978 asistimos a la reivindicación realizada por Patrocinio de las Heras (2000), cuyo propósito era eliminar el término beneficencia pública del texto constitucional. A estas reivindicaciones se sumaron las realizadas por otras profesionales como Karmentxu Marín en 1976 («Asistentes Sociales: una profesión para tomar en serio») y la propia Patricinio de las Heras en 1978 («Grave carencia de una correcta planificación de la acción social») (Llovet y Usieto, 1990).

A su vez, y como veremos en el caso de la Escuela de Asistentes Sociales de Pamplona, este es un período de incertidumbre para las escuelas de Trabajo Social dependientes de la Iglesia, ya que muchas de ellas se ven abocadas a cerrar. Pese a todo, es un período en el que se le da muy poca importancia a asignaturas propias del Trabajo Social en los planes de estudio (Brezmes, 2008).

Se evidencia que, desde el punto de vista axiológico, es el momento en el que las y los profesionales empiezan a tomar conciencia de un espacio ético propio, destacando valores como la profesionalidad, la entrega y el sentido de responsabilidad, así como la explicitación de la toma de conciencia del dilema ético entre la tarea de promoción de la persona usuaria y la labor de control del profesional (Vázquez, 1971).

Así, aunque continuase habiendo profesionales con un marcado carácter religioso, en este período comienzan a proliferar las y los que consideran que la ética profesional debe desvincularse definitivamente de la moral religiosa (Vázquez, 1971; Brezmes, 2008).

\subsection{Periodo de principios éticos, burocratización y reivindicaciones (1980-2002)}

El fenómeno que predomina en el Trabajo Social entre 1980 y 2002 es la burocratización y el consiguiente empobrecimiento de la disciplina-profesión, así como las reivindicaciones de las y los profesionales, entre las que preponderan las posturas de izquierdas y la no identificación de la profesión con los valores de Iglesia (Idareta, Úriz y Viscarret, 2016).

La profesión asiste también a contradicciones fundamentales en esta época: trabaja por adaptar a la persona a la realidad social generadora de los problemas sociales; no defiende lo público, sino que lo critica y apuesta por la iniciativa privada; las profesionales del Trabajo Social, aunque busquen un estatus científico, no se preocupan por producir un discurso disciplinar propio; $y$, finalmente, se desconoce cómo promover la autonomía de la persona usuaria mientras que a las y los profesionales se les asignan labores de control (Brezmes, 
2008) ${ }^{3}$. Curiosamente este último es un dilema ético que también fue citado por Vázquez (1971) y por Sarasa (1993).

\section{Periodización de los valores éticos en el Trabajo Social en Navarra}

Hemos establecido dos períodos entre 1960 y 1985: el período moralizador (1960-1980) y el período de principios éticos, burocratización y reivindicaciones (1980-1985). El primero de ellos engarza con el declive del período moralizador que se origina a nivel nacional, al que en Navarra se le da una prolongada continuidad. El segundo coincide con el período del mismo nombre que acontece a nivel nacional en 1980 de la mano de la incipiente secularización del Trabajo Social en Navarra.

Al primer período lo hemos denominado moralizador porque la tendencia del Trabajo Social durante este período es bastante paternalista, velando mucho más por el bienestar de la persona usuaria que por la promoción de su autonomía o por la prevención de los problemas sociales. En el segundo período se originan las reivindicaciones de las y los profesionales y los servicios sociales comienzan a padecer los efectos de la burocratización, sumándose por ello a la reflexión que la profesión realiza a nivel nacional sobre la importancia de los principios éticos. De ahí su nombre: período de principios éticos, burocratización y reivindicaciones.

\subsection{Período moralizador (1960-1980)}

Debido al desproporcionado e inesperado crecimiento de la población de Pamplona originado entre 1950 y 1970 por los movimientos migratorios de zonas rurales hacia la capital para acceder a la incipiente industria, se evidenciará una importante falta de acondicionamiento de la ciudad que traerá como consecuencia diversos focos de marginalidad (Campo y Celaya, 1981).

Frente a tales problemas sociales se contempló la necesidad de crear nuevas instituciones y de formar nuevos profesionales para poder responder organizadamente a sus demandas y carencias. Así, en un contexto en el que se originará la proletarización de la población, la aparición de una clase empresarial y la renovación de la Iglesia Católica a partir del Concilio Vaticano II, en septiembre

3. Compartimos con Brezmes (2008) que el común denominador de estos períodos es la burocratización del Trabajo Social y añadiremos que esta progresiva tecnificación ha dado lugar a una intervención social excesivamente instrumentalizada (Idareta y Ballestero, 2013). No obstante, creemos que no es válido señalar todas estas contradicciones y renegar de la perspectiva ética gracias a la cual han podido ser identificadas. 
de 1960 se abrirá la Escuela de Asistentes Sociales «San Vicente de Paúl» de Pamplona.

La Escuela surge al amparo del Arzobispado de Pamplona. Comenzará su andadura en los locales de la Providencia, siendo José María Díaz Madoz delegado episcopal. La dirección de la Escuela corrió a cargo de las Hijas de la Caridad (Sor María Ángeles del Amo, Sor María Rosa Sánchez, Sor María Ángeles Brun y Sor Josefina Herrero) hasta el curso 1973-1974.

La primera promoción se graduará en 1963 y la conformaron una treintena de alumnas (Romero, 2015). Estas promociones estaban compuestas exclusivamente por mujeres de entre 22 y 25 años, algunas de ellas religiosas de la misma Congregación, que destacaban por su vocación y su compromiso social. Los primeros hombres no se matricularon hasta el curso 1974-1975 (Campo y Celaya, 1981).

Estas primeras promociones se agruparán conformando la Agrupación de Asistentes Sociales «Santa María La Real», que aparece registrada en el Libro de Registro de Asociaciones desde el 13 de abril de 1965. Sus principales objetivos serán: elevar la formación técnica, moral y religiosa de sus miembros y estudiar las cuestiones doctrinales, científicas y prácticas del Servicio Social, mediante contactos nacionales e internacionales.

Por otra parte, aunque en otros planes de estudios de la época hallemos las asignaturas sobre moral profesional vinculada con la de formación religiosa, en el plan de estudios de la Escuela de Pamplona del curso 1962-1963 la asignatura Moral Profesional se imparte en el tercer curso, probablemente muy condicionada por preceptos religiosos anteriormente impartidos en las asignaturas de Dogma y Sagrada Escritura del primer curso o Doctrina Social de la Iglesia del segundo curso (Romero, 2015).

En este sentido, no se debe olvidar que las Escuelas de Asistentes Sociales «San Vicente de Paúl» tenían como objetivo principal la formación de las religiosas, así como de las que aspiraban a serlo. De ahí la vinculación existente entre formación religiosa y moral profesional.

Por ello, del mismo modo que sucediese a nivel nacional (Idareta, Úriz y Viscarret, 2016), aunque estas asignaturas poseyesen una marcada impronta religiosa, no se debe perder de vista la importancia que se le otorgaba a la formación en ética profesional (Molina, 1990, 1994; Sarasa, 1993). De hecho, el trato humanizador y el fuerte sentido ético aparecen como rasgos distintivos comunes de las primeras promociones de asistentes sociales y de sus formadores, así como elemento esencial en la formación recibida en la Escuela de Pamplona (Molina, 1990, 1994). 
El carácter religioso de las actividades profesionales de las primeras asistentes sociales lo hallamos en algunos artículos de prensa de la época (Diario de Navarra del 09/08/1963 y ABC del 11/10/1970), en los que son consideradas como instrumentos de la Iglesia técnicamente capacitadas para dispensar, con vocación de servicio, la caridad cristiana y el cuidado pertinente a las y los feligreses.

Se destaca que la formación universitaria es innecesaria puesto que la asistencia social es una técnica aplicada cuya finalidad es resolver con inmediatez los problemas sociales del momento (económicos, escolares, de vivienda, de trabajo, de salud, etc.). Por todo ello, la asistente social era considerada como «cabeza que piensa, corazón que obedece, y manos siempre listas a la acción» (Diario de Navarra, 09/08/1963) o como «voluntaria casi sin preparación» con «deseo de hacer algo por los demás» (Unidad, 14/02/70). En este sentido, cabría un estudio a fondo de la incidencia del principialismo ético en el Trabajo Social en Navarra.

Todo ello evidencia que el Trabajo Social en Navarra, del mismo modo que en el resto de España, se comenzó a ejercer sin un método concreto y sin cuestionar el discurso hegemónico imperante (Romero, 2015; Idareta, Úriz y Viscarret, 2016), pudiendo compensar esta importante falta de orientación técnica con una fuerte orientación ética. Gran parte de los testimonios de la época apuntan a que los valores éticos fueron fundamentales para el ejercicio profesional ante la ausencia de método específico de Trabajo Social (Hörler, 1961; Llovet y Usieto, 1990).

En el caso de la Escuela de Pamplona, en el Estatuto de la Escuela de 1977 queda patente que es competencia de la Junta de Promoción «orientar la Educación Religiosa del Alumnado» (Artículo 2. ${ }^{\circ}$, Base Tercera) y que el Director de la Escuela se hará cargo de «el nombramiento y separación del Profesorado, tanto en las áreas de docencia teórica como práctica, excepto el personal de educación religiosa» (Artículo 1. ${ }^{\circ}$, Base Sexta). Todo ello revela la dependencia institucional que la Escuela seguía manteniendo con la Iglesia.

Entre el 26 y 30 de septiembre de ese mismo año, se celebraron las III Jornadas Nacionales de Asistentes Sociales en Pamplona (concretamente en la Ciudad Deportiva de Amaya), en las que se ratificará el cambio de denominación de la profesión (Trabajo Social en vez de Asistencia Social) ya propuesto desde el Congreso de 1968.

No obstante, como reconoce Brezmes (2008), la modificación de la denominación de la profesión no originó ningún tipo de cambio sustancial en la misma. Desde una perspectiva ética, concretamente en Navarra, los valores predominantes eran los religiosos, pese a que, del mismo modo que otras 
comunidades autónomas, se adscribiesen al Código de Ética de la FITS. Se pretendía paliar los problemas sin contemplar la prevención de los mismos o la promoción de la autonomía de la persona usuaria, predominando un enfoque paternalista, así como una atención individual o familiar.

La Constitución Española de 1978, servirá de marco jurídico hacia la democracia al definir a España como un «Estado social y democrático de Derecho», dando a los españoles el status de «ciudadanos con derechos». Comenzará un proceso legislativo que derogará normas obsoletas e implantará otras que se ajusten al nuevo régimen democrático y de Derecho (Brezmes, 2008). Este proceso legislativo, junto con la creación del sistema público de Servicios Sociales, uniformizará y sincronizará las evoluciones de las distintas realidades de las comunidades autónomas en materia de asistencia social.

\subsection{Principios éticos, burocratización y reivindicaciones}

La secularización del Trabajo Social en Navarra comienza a finales de los años 70 (Campo y Celaya, 1981) con el abandono paulatino de prácticas voluntaristas y la consiguiente profesionalización de la intervención social. Pero en 1980 la Escuela aspira a depender no tanto del Arzobispado como de la Diputación Foral. Todo ello porque a finales de los 70, el Arzobispado amenaza con el cierre de la Escuela debido a motivos económico y a los cambios sociales.

En los años 80, la Diputación Foral asumirá las competencias de los Servicios Sociales mediante acuerdos con la Administración Central, dando lugar a la creación del Organismo Autónomo de Servicios de Asistencia Social en 1981. Por su parte, el Ayuntamiento de Pamplona también impulsará los Servicios Sociales contratando a asistentes sociales para modernizar la intervención y pasar de un sistema de beneficencia paternalista a uno basado en el Estado de Bienestar y la justicia social.

En este sentido, la Ley Foral de Servicios Sociales de 1983 recogerá los principios y las áreas fundamentales de los servicios, políticas y competencias de la Diputación Foral. Este acondicionamiento de los Servicios Sociales de la Comunidad Foral incrementará los servicios ofrecidos pero redundará en una disminución de la atención directa y de un consiguiente incremento del grado de burocratización de la profesión (Idareta y Úriz, 2016).

La Escuela de Asistentes Sociales «San Vicente de Paúl» desaparece en el curso 1984-1985, el mismo en el que se cumplían 25 años de su creación, para dejar paso a la Escuela Universitaria del Gobierno de Navarra. La Escuela no pudo asumir los requisitos para transformarse en Escuela Universitaria de Trabajo Social porque carecía de los medios económicos y de Universidad pública a la que adscribirse. 
Así, en 1984 comienzan a darse los pasos definitivos para que el Trabajo Social adquiera rango universitario en Navarra, adscribiéndose a la Universidad de Zaragoza hasta el año 1991. Momento en el que pasa a incorporarse a la Universidad Pública de Navarra (UPNA). La primera promoción de trabajadoras y trabajadores sociales de la UPNA se diplomará el año 1993, tres décadas más tarde que la primera de asistentes sociales.

En definitiva, es un período en el que el foco de atención se centra en la consolidación de los servicios sociales en Navarra, en la toma de conciencia de los efectos de la burocratización de los mismos, en reflexionar sobre la necesidad de un Código de Ética a nivel nacional, así como en la incorporación de la profesión a la universidad.

\section{Conclusiones}

Desde una perspectiva ética, el caso del Trabajo Social de Navarra difiere sustancialmente de la evolución seguida en el conjunto de España. Mientras que a nivel estatal entre 1960 y 1985 se delimitan claramente tres períodos éticos (período moralizador, período de valores y período de principios éticos, burocratización y reivindicaciones) (Idareta, Úriz y Viscarret, 2016), en Navarra únicamente se identifican dos (período moralizador y período de principios éticos, reivindicaciones y burocratización).

A tenor de la bibliografía analizada, no se han hallado evidencias que permitan delimitar un período de valores, entendiendo por tal un período en el que las y los profesionales tomen conciencia y recapaciten sobre los valores éticos con los que creen que deben identificarse.

En este sentido, tras el período moralizador, se inicia el período de principios éticos, burocratización y reivindicaciones, por lo que habrá que estudiar las consecuencias e implicaciones que haya podido traer para el ejercicio profesional en Navarra la ausencia de período de valores, así como un período moralizar tan prolongado.

La fuerte influencia de la Iglesia en Navarra, unida a la dependencia institucional que mantuvo la Escuela hasta los años 80 , pudieron ser algunos de los motivos principales de la ausencia de un período de valores éticos claramente delimitado en el que se reflexionase sobre la conducta moral de las y los profesionales desde una perspectiva estrictamente ética.

Por todo ello, podemos afirmar que mientras que en gran parte de España el proceso de secularización de las escuelas de asistentes sociales se inicia a principios de los 60, en Navarra comienza durante los últimos años de los 70 y primeros de los 80 . Por tanto, en Navarra el período moralizador se torna 
veinte años más largo, ya que en el resto del país, entre 1960 y 1980, se desarrolla el período de valores.

El incipiente proceso de secularización de la Escuela en Navarra, que apostaba por una dependencia de la Diputación Foral y la adscripción a la Universidad, centraron el foco de gran parte de las preocupaciones y las reivindicaciones de las y los profesionales del Trabajo Social hasta bien entrada la década de los 80 .

Década durante la cual, en sincronía con el Trabajo Social nacional, el Trabajo Social en Navarra comenzó a padecer los efectos de una fuerte burocratización. Desde una perspectiva ética, es a partir de entonces cuando el Trabajo Social en Navarra comienza a seguir la misma evolución que en el resto de España, sumándose a las reflexiones sobre las consecuencias de la alta burocratización en los servicios sociales y sobre la importancia de un código deontológico a nivel estatal.

\section{Bibliografía}

Bermejo, FJ. (2002). Ética de las profesiones. Bilbao: Desclée Brower.

BREZMES, M. (2008). El Trabajo Social en España. Murcia: Editum.

CAMPO, M.A. Y CELAYA, T. (1981). Los trabajadores sociales-asistentes sociales y el futuro de Navarra: perspectivas de una profesión. Pamplona: Escuela de Asistentes Sociales San Vicente de Paúl.

Colomer, M. (1968). «Estatus profesional y deontología», en Memoria del I Congreso Nacional de Asistentes Sociales (pp. 65-71). Madrid: FEDAAS.

DE LAS HERAS, P. (2000). «La construcción histórica de la profesión», en II Foro de Trabajo Social: Pasado, presente y futuro del Trabajo Social (pp. 15-82). Madrid: Universidad de Comillas.

Díaz GARCíA, E. (1985). La filosofía social del krausismo español. Madrid: EDICUSA. DomínGUEZ, D. (2011). El estoicismo como moral en Vives, el Broncese y Quevedo. Ingenium. Revista de historia del pensamiento moderno, 5, 105-131.

EsTRUCH, J. y GÜELL, A. (1976). Sociología de una profesión: los asistentes sociales. Barcelona: Península.

Fernández de Henestrosa, M. (1946). Algunas ideas sobre la caridad organizada (para uso de las visitadoras). Madrid: Consejo Superior de Protección de Menores. Sección de Asistencia Social.

FERnÁNDEZ, A. y CoRvalán, H. (2001). Influencia del krausismo en el Río de Plata. Frónesis, 8 (2), 83-95.

GANIVET, A. (1998). Idearium español. Madrid: Biblioteca Nueva.

GIL PAREJO, M. (2004). El protagonismo de la organización colegial en el desarrollo del Trabajo Social en España. Madrid: Siglo XXI. 
GuilLÉN, E. (1993). La burocratización de Trabajo Social en la Intervención Social. Cuadernos de Trabajo Social, 6, 181-193.

GutiérRez ResA, A. (2010) Aplicación de valores en los servicios sociales comunitarios. Praxis sociológica, 14, 115-143.

Heylen, V.L. (1960). Código moral del Servicio Social. Madrid: Cáritas española Centro de Estudios de Sociología Aplicada - Instituto Católico de Estudios Sociales.

HÖrLer, E. (1961). Origen y objeto del Servicio Social. Misión de las Asistentes Sociales. Madrid: Consejo Superior de Investigaciones Científicas.

IDARETA, F., ÚRIZ, M. J. y VisCARRET, J.J. (2016, en prensa). 150 años de historia de la Ética del Trabajo Social: periodización de sus valores éticos. Cuadernos de Trabajo Social.

IDARETA, F. y BAllestero, A. (2013). La evolución del compromiso ético de los trabajadores sociales (1869-2013). Zerbitzuan, 54, 147-155.

LacalzadA, M.'J. (2012). Concepción Arenal: mentalidad y proyección social. Zaragoza: Prensas Universitarias de Zaragoza.

Llovet, J.J. y Usieto, R. (1990). Los trabajadores sociales. De la crisis de identidad a la profesionalización. Madrid: Popular.

Molina, M. ${ }^{a}$ V. (1990). Las Escuelas de Trabajo Social en España (1932-1983). Colección Tesis Doctorales, 127/90. Madrid: Universidad Complutense de Madrid.

MolinA, M. ${ }^{2}$ V. (1994). Las enseñanzas del Trabajo Social España, 1932-1983. Estudio socio-educativo, Madrid: Universidad Pontificia Comillas de Madrid.

Motтo, A. (2011). San Vicente de Paúl y la corriente renano-flamenca. Vicencianos. http://vicencianos.org/san-vicente-de-paul-y-la-corriente-renano-flamenca/

PÉREZ, M.E. (2002). Revisión de las ideas morales y políticas de Concepción Arenal. Tesis doctoral. Madrid: Universidad Complutense de Madrid.

Romero, I. (2015). La profesionalización del Trabajo Social: Los inicios en Navarra en la segunda mitad del siglo XX (Trabajo Fin de Máster). Pamplona: Universidad Pública de Navarra. Extraído de: http://academica-e.unavarra.es/ handle/2454/19413

Roviralta, R. (1937). Los problemas de la Asistencia Social en la Nueva España [s.l.: s.n.]

SARASA, S. (1993). El servicio de lo social. Madrid: INSERSO.

VÁzQUEZ, J.M. (Dir.) (1971). Situación del Servicio Social en España. Madrid: Instituto de Sociología Aplicada.

ZAMbrano, M. (1992). El pensamiento vivo de Séneca. Madrid: Cátedra.

Zambrano, M. (1996). Pensamiento y poesía en la vida española. Madrid: Endymion. 